\title{
Anabases
}

ANABASES Traditions et réceptions de l'Antiquité

1 | 2005

Varia

\section{La papyrologie documentaire grecque en 2005 : bilan et perspectives}

\section{Bernard Legras}

\section{(2) OpenEdition}

1 Journals

Édition électronique

URL : http://journals.openedition.org/anabases/1480

DOI : 10.4000/anabases. 1480

ISSN : 2256-9421

Éditeur

E.R.A.S.M.E.

\section{Édition imprimée}

Date de publication : 1 mars 2005

Pagination : 215-231

ISSN : 1774-4296

\section{Référence électronique}

Bernard Legras, «La papyrologie documentaire grecque en 2005 : bilan et perspectives », Anabases

[En ligne], 1 | 2005, mis en ligne le 01 septembre 2011, consulté le 20 octobre 2019. URL : http:// journals.openedition.org/anabases/1480 ; DOI : 10.4000/anabases.1480 
Anabases 1 (2005), p. 215-231

\section{La papyrologie documentaire grecque en 2005 : bilan et perspectives}

BERNARD LEGRAS

LES PREMIÈRES ANNÉES du troisième millénaire ont été particulièrement riches en publications dressant un bilan des sources textuelles grecques concernant l'Égypte grecque et romaine. En 2001 paraissait une version refondue de la Checklist des éditions de papyrus, d'ostraca et de tablettes ${ }^{1}$, qui permet de prendre la mesure du développement des publications issues de collections que le World Wide paru en 2000 offre de connaitre plus précisément ${ }^{2}$. En 2002, Étienne Bernand faisait paraître un nouvel état du corpus des inscriptions grecques d'Égypte et de Nubie, qui signifiait l'importance de nouvelles publications de la chôra et d'Alexandrie ${ }^{3}$. La même année Luz María García Fleitas et Germán Santana Henríquez tentaient un recensement de l'image de l'Égypte chez les historiens grecs du Ve siècle av. n. è. au IV ${ }^{\mathrm{e}}$ siècle de n. è. ${ }^{4}$

Ces trois publications ont en commun de vouloir offrir un accès global, aux débutants comme aux chercheurs chevronnés, vers des sources textuelles grecques et

1 J.F. OATES, R.S. BAGNALL, S.J. CLACKSON, A.A. O’BRIEN, J.D. SOSIN, T.G.W ILFONG, K.A.W ORP, Checklist of Editions of Greek, Latin, Demotic and Coptic Papyri, Ostraca and Tablets, Fifth Edition, Oakville, Connecticut, 2001 (Bulletin of the American Society of Papyrologists. Supplements 9):

<http://www.scriptorium.lib.duke.edu/papyrus/texts/clist.html>

2 W. Clarysse et H. Verreth (éd.), Papyrus Collections World Wide (9-10 mars 2000, Bruxelles-Louvain), Bruxelles, 2000 (Koninklijke Vlaamse Academie van Belgie voor Wetenschappen en Kunsten): <http://milennium.ars.kuleuven.ac.be//lhp>

3 É. BERNAND, "État du corpus des $I G$ d'Égypte et de Nubie", Zeitschrift für Papyrologie und Epigraphik 139 (2002), p. 119-126. Il faudrait ajouter au programme des futures publications des déserts occidental et oriental. Cet article donne la bibliographie d'André et d'Étienne Bernand.

4 L.M. GARCÍA FLEITAS et G. SANTANA HENRÍQUEZ, La imagen de Egipto en los fragmentos de los historiadores griegos. Una primera aproximación, Universidad de Las Palmas de Gran Canaria, 2002. 
latines qui sont complémentaires. La parution en 2000 du Manuel d'études démotiques de Friedhelm Hoffmann, venant après celui de Marc Depauw paru en 1997, permettait parallèlement au chercheur familier des sources grecques et latines de s'initier plus facilement au monde des sources démotiques 5 .

Ces publications interviennent au moment où la papyrologie, cette science toujours jeune, célèbre son premier centenaire. Elle est en effet consciente de sa spécificité au début du XX ${ }^{\mathrm{e}}$ siècle ${ }^{6}$. Si le néologisme "papyrology» est forgé en 1898 par les «Dioscures d'Oxford», Bernard Grenfell et Arthur Hunt, avant d'être introduit en allemand (Papyruskunde/Papyrusforschung), en italien (Papirologia), en français et en néerlandais (Papyrologie) en 1901, il faut attendre 1908 pour voir l'apparition de l'adjectif "papyrological», et 1922 pour voir la naissance du substantif "papyrologist» 7 .

Nous voudrions ici rappeler la diversité des spécialisations papyrologiques, et présenter certains enjeux actuels de la papyrologie documentaire.

\section{La diversité des spécialisations papyrologiques}

Jean Bingen rappelle très opportunément qu' «il y a presque autant de papyrologies qu'il y a de papyrologues, et ils sont nombreux" ${ }^{8}$. L'Association Internationale de Papyrologues comprenant des centaines de membres disséminés de l'Europe aux Amériques, de l'Australie au Japon, il est nécessaire de regrouper ces idiosyncrasies en thématiques simples. Il semble que trois critères de différentiation puissent être définis, le support matériel de l'écriture, la définition du "papyrologue» et les champs disciplinaires papyrologiques.

5 F. HOFFMANN, Ägypten: Kultur und Lebenswelt in griechisch-römischen Zeit. Eine Darstellung nach den demotischen Quellen, Berlin, 2000; M.D EPAUW, A Companion to Demotic Studies, Bruxelles, 1997.

6 Sur cette «jeunesse» persistante de la discipline, cf. W. SCHUBART, Einführung in die Papyruskunde, Berlin, 1918, p. 1: «Die Papyruskunde ist eine junge Wissenschaft, deren Anfänge noch nicht weit zurückliegen»; O.M ONTEVECCHI, La papirologia, $2^{\mathrm{e}}$ éd., Milan, 1988, p. 3: «una disciplina ancor giovane»; aussi H.-A. RUPPRECHT, Kleine Einführung in die Papyruskunde, Darmstadt, 1993, p. 1: "Die Papyrologie oder Papyruskunde ist eine junge Wissenschaft, die eine allgemein akzeptierte Definition ihres Gebietes noch nicht gefunden hat».

7 Cf. K.A. WORP, Keerpunten in de Papyrologie [Tournants dans la papyrologie], Leyde, Universiteit Leiden, 2003, qui insiste sur la papyrologie aux Pays-Bas.

8 Cité par J.A. STRAUS, Initiation à la papyrologie documentaire, $5^{\mathrm{e}}$ édition revue, dactyl., Liège, Année universitaire 2000-2001, p. 14. Cf. aussi H.-A. RUPPRECHT, Einführung, p. 1: "...so daß man sagen konnte, es gebe so viele Definitionen von Papyrologie wie Papyrologen selbst». 


\subsection{Le support matériel}

Étymologiquement, la papyrologie est la discipline qui étudie les papyrus en tant que support de l'écriture et les textes qu'ils portent. Mais cette définition étroite est supplantée par une définition large qui l'institue comme la discipline étudiant les textes antiques venus d'Égypte écrits à l'encre, qu'ils soient écrits sur papyrus, sur tessons (ostraca), tablettes de bois, cuir, parchemin, lin, etc. Cependant l'étude des tablettes de bois incisées au stylet relève aussi, au sens large, de la papyrologie.

Un consensus semble s'être établi autour de la définition large de la papyrologie.

Ces textes sont en langue égyptienne (hiératique, démotique, copte), araméenne, grecque, latine ou arabe. Certains sont bilingues. La multiplication des publications de papyrus d'époque ptolémaïque et romaine en égyptien, en particulier en démotique, a rendu nécessaire l'utilisation d'un adjectif "grecque», «latine» ou "démotique», un impératif qui n’a pas été perçu jusqu’à une époque récente où le mot "papyrologie» sans précision signifiait l'étude des papyrus grecs (et accessoirement latins).

\subsection{Qu'est-ce qu'un papyrologue?}

Depuis la naissance de la papyrologie, deux définitions cohabitent. La première restreint la qualification de papyrologue à celui qui déchiffre, édite, traduit et commente les textes conservés sur les supports matériels englobés par la papyrologie. C’est le point de vue défendu par André Bataille qui estime que «le papyrologue est d'abord un paléographe», car "l'unité de la discipline (repose sur le fait) qu'on écrivait de même façon sur ostracon ou tablette de bois que sur papyrus et dans le reste du monde grec comme en Égypte». "Le vrai travail papyrologique [...] se fait sur les originaux» 9. Il s'accomplit "depuis la fouille jusqu'à la publication». La seconde définition l'élargit en considérant que toute personne qui fonde ses recherches - en partie ou en totalité - sur ce type de sources peut être considéré comme un "papyrologue». Il existe ainsi des papyrologues "éditeurs" et des papyrologues «non éditeurs». La définition la plus large est retenue par l'Association Internationale de Papyrologues, fondée en 1932 à Bruxelles, dont l'annuaire fait apparaître des chercheurs qui appartiennent à des domaines scientifiques très divers: archéologues, biblistes, égyptologues, historiens des mondes grec et romain, juristes, patristiciens, philologues classiques, etc. Les Congrès internationaux de Papyrologie, qui se tiennent tous les trois ans (le dernier, le XXIVe, s'est tenu en août 2004 à Helsinki, le prochain aura lieu à en 2007 à Ann

9 A. BATAILle, "Papyrologie", in L'Histoire et ses méthodes, Encyclopédie de la Pléiade, C.S AMARAN éd., Paris, 1967, p. 510-511, 522-523. 
Arbor) rassemble donc des communications dans de multiples domaines de l'activité scientifique ${ }^{10}$.

L'activité des papyrologues n'est pas circonscrite à l'Égypte, ni à l'Antiquité.

Si l'immense majorité des papyrus a été retrouvée en Égypte, ceux issus d'autres régions occupent une part significative. Les premiers papyrus retrouvés à l'époque moderne, en 1752, sont ceux d'Herculanum, qui proviennent de la "villa des papyrus» ensevelie en 79 de n. è. sous les laves du Vésuve. Le plus ancien papyrus grec actuellement connu (IVe siècle av. n. è.), découvert en 1962, est issu de Dervéni en Macédoine grecque. Des papyrus grecs, latins, araméens, nabatéens et syriaques proviennent du Proche et du Moyen-Orient, Doura Europos (Moyen Euphrate), Avroman (Kurdistan turc), Israël/Palestine (Hibet el-Mird, Massada, Nahal Hever, Nessana, Qumran), de Jordanie (Pétra) et de Nubie (Qasr Ibrim). Le plus ancien papyrus trouvé hors d'Égypte, provient de Palestine (P. Murabba'at 17). Ce papyrus écrit en hébreu date d'environ de 750 av. n. è.

Les conditions climatiques de l'Égypte, la sécheresse expliquent sa conservation à travers les siècles et les millénaires. Hors d'Égypte, le papyrus ne se conserve que dans un environnement comparable (Néguev, désert de Syrie) ou sous forme "carbonisée» (Dervéni, Pétra). Des découvertes attestent que l'espace géographique de son usage dans le monde grec et romain antique était plus large que la géographie des trouvailles: le papyrus mis au jour en 1963 par un archéologue en Roumanie, à Callatis, s'est ainsi détruit peu après sa découverte.

La papyrologie ne concerne pas le seul monde antique, mais aussi la période historique qui lui fait suite, le Moyen Âge, puisque le dernier usage connu du papyrus (un texte arabe d'Égypte) date de 1378. La moisson papyrologique est particulièrement riche pour l'Égypte byzantine. Mais un débat existe sur l'époque où s'achève "l'Antiquité» 11. En Occident, le papyrus n'a cédé - comme en Orient - que progressivement face au parchemin. Les papyrus font partie des importations du port de Marseille du VI ${ }^{\mathrm{e}}$ au VIII ${ }^{\mathrm{e}}$ siècle. Il est utilisé en France jusqu'en 787, et par la chancellerie papale jusqu'en 1057. Le "papyrus» ne survit plus alors que dans

10 La division en sessions lors du XXIVe Congrès international de papyrologues à Helsinki (1-7/8/2004) permet de rendre compte des spécialisations: A/ Collections, preservation and conservation, B/ Literary and semi-literary papyri, C/ Papyri and biblical studies, D/ Religion and magic, E/Juristic papyrology, F/ Onomastics and prosopography, G/Linguistics, H/ Social and cultural history, I/ Economy, J/ Topography, K/ Material culture, L/ Palaeography, M/ Papyri of the Near East, N/ Herculanensia, O/ Papyrology and Egyptology (Demotic, Coptic, Arabic), P/ Digital technology.

11 A. GIARDINA, "Egitto bizantino o tardo antico? Problemi della terminologia e della periodizzazione", in Egitto e storia antica dall'ellenismo all'età araba. Bilancio di un confronto. Atti del colloquio internazionale (Bologna, 31 agosto-2 settembre 1987), Bologne, 1989, p. 89-103. 
le mot "papier» qui est formé sur ce terme latin lui-même issu du grec qui emprunte le mot à l'égyptien $p 3-p r(p a-p o r){ }^{12}$.

\subsection{Les champs disciplinaires papyrologiques}

La diversité des textes papyrologiques pose la question de l'unité de la discipline, une question étroitement liée à son statut parmi les sciences de l'Antiquité, l'Altertumswissenschaft de la recherche allemande. S'agit-il d'une "discipline fondamentale» (Grundlagenwissenschaft), d'une "science auxiliaire» (Hilfswissenschaft) ou d'une "branche autonome des sciences de l'Antiquité classique» (eigenständiger Zweig in die Klassische Altertumswissenschaft) ${ }^{13}$ ?

L'unité de la discipline est défendue par certains, en particulier Marcello Gigante ou Eric G. Turner ${ }^{14}$. Mais la spécialisation des papyrologues est une réalité qui est apparue très tôt, en fonction de ces grands champs du savoir que sont l'histoire, le droit et la littérature. Les deux premiers constituent la papyrologie documentaire, la troisième, la papyrologie littéraire ${ }^{15}$.

Le premier Manuel de Papyrologie documentaire, paru en Allemagne, en $1912^{16}$, est de fait confié à deux auteurs, d'une part l'historien Ulrich Wilcken (1862-1944), qui écrit les deux volumes consacrés à la partie historique (Historischer Teil), et d'autre part le juriste Ludwig Mitteis (1859-1921) qui se charge de la partie juridique (Juristischer Teil). L'introduction méthodologique et programmatique d'Ulrich Wilcken définit clairement cet ouvrage fondateur comme une présentation et une anthologie de papyrus documentaires (Papyrusurkunden), qui laisse de côté les papyrus littéraires relevant de la littérature grecque et latine. Seuls les aspects formels de ces papyrus littéraires sont

12 J. VERGOTE, "L'étymologie du mot "papyrus", Chronique d'Égypte 60 (1985), p. 393397: il signifie "celui de la maison", "celui du domaine (royal)" avec le sens de "plante de l'administration" qui révèle son caractère de monopole royal à l'époque pharaonique (et ptolémaïque).

13 Cf. H.-A. RUPPRECHT, Einführung, p. 1.

14 M. GigAnTE, "Per l'unità della scienza papirologica", in Atti del XVII Congresso Internazionale di Papirologia (19-26 maggio 1983), I, Naples, 1984, p. 5-28; E.G. TURNER, Greek Papyri. An Introduction, $2^{\mathrm{e}}$ éd., Oxford, 1980, p.VII.

15 Ce partage existe depuis les débuts de la science papyrologique. La "centrale d'achat" (Deutsche Papyrus-Kartell, DPK) constituée par les instituts allemands (dont la Kaiser Wilhelms-Universität, aujourd'hui l'Université Marc Bloch, de Strasbourg), en 1902, pour gérer la concurrence et coordonner les achats en Égypte est organisée en deux sections, documentaire (Abteilung $A$ ) et littéraire (Abteilung B).

16 U. WILCKEN, Grundzüge und Chrestomathie der Papyruskunde, Historischer Teil, 2 vol., Leipzig-Berlin, 1912; L. MITTEIS, Grundzüge und Chrestomathie der Papyruskunde, Juristischer Teil, 2 vol., Leipzig-Berlin, 1912. 
à prendre en compte par les papyrologues: l'écriture, le format, les données bibliologiques.

Les papyrologies documentaire et littéraire peuvent être divisées en deux grandes sous-disciplines.

La papyrologie historique offre aux historiens de l'Égypte grecque, romaine et byzantine, ainsi qu'aux spécialistes d'autres régions du monde antique pourvues $\mathrm{de}$ documentation papyrologique, des types de documents couvrant tous les aspects de la vie antique. Les thèmes des douze chapitres des Grundzüge d'Ulrich Wilcken en offrent une vision synthétique ${ }^{17}$. Le plaidoyer de Roger Bagnall offre des exemples emblématiques du rôle de la documentation papyrologique pour écrire l'histoire des mondes antiques ${ }^{18}$. L'historien ne peut cependant se limiter à ce type de documentation pour construire une histoire globale. Les sources papyrologiques doivent être complétées avec les données des sources littéraires, de l'épigraphie, de l'archéologie, de la numismatique etc. Il doit - comme l'écrit Claude Nicolet «reconstituer à travers cette diversité (des disciplines ou des sous-disciplines), un processus unitaire» 19.

La papyrologie juridique fonde sa spécificité sur les papyrus conservant des cas de la pratique, des traités ou des codes relevant des droits de l'Antiquité ${ }^{20}$. Les juristes et les historiens du droit grec et hellénistique, ainsi que du droit romain, ont vite pris la mesure de l'abondance de données juridiques souvent exceptionnelles, permettant en particulier de s'intéresser à la problématique des antécédents juridiques du droit grec des cités dans l'Égypte grecque, de la survie de ce droit grec et hellénistique dans l'Égypte romaine, et de l'éventuelle originalité de la

17 I/ Allgemeine historische Grundzüge, II/ Religion und Kultus, III/ Die Erziehung, IV/ Die Finanz-Ressorts. Ihre Organe und Kassen, V/ Das Steuerwesen, VI/ Industrie und Handel, VII/ Die Bodenwirtschaft, VIII/ Fronarbeiten und Liturgien, IX/ Das Verpflegungswesen, X/ Das Post-und Transportwesen, XI/ Militär und Polizei, XII/ Aus dem Volksleben.

18 R.S. BAGNALL, Reading Papyri, Writing Ancient History, Londres-New York, 1995.

19 C. NiCOLET, La mémoire perdue. À la recherche des archives oubliées, publiques et privées, de la Rome antique, Paris, 1994, p. VIII-IX, qui met en avant le risque de la spécialisation "à outrance», qui conduit à ne prendre en compte qu'un support: "Ce parti-pris fâcheux est malheureusement la règle pour l'étude de l'Antiquité, et a déterminé en gros la division de nos sous-disciplines, philologie (codicologie et "manuscrits" littéraires), épigraphie (le "support dur", en gros) et papyrologie, voire numismatique».

20 Cf. A. BISCARDI et E. CANTARELLA, Corso di papirologia giuridica, Milan, 1966; J.M ÉLĖZE MODRZEJEWSKI, Loi et coutume dans l'Égypte grecque et romaine. Recherches sur les facteurs de formation du droit privé en Égypte aux temps des Lagides et sous la domination romaine (dactyl.), Paris, 1970; L. MIGLIARDI ZINGALE, Introduzione allo studio della papirologia giuridica, $2^{\mathrm{e}}$ éd., Turin, 1994; H.-J. WOLFF, Vorlesungen über juristische Papyruskunde, Berlin, 1998; G. PURPURA, Diritto, papiri e scrittura, $2^{\mathrm{e}}$ éd., Turin, 1999. 
province romaine d'Égypte dans l'Empire mondial des Romains ${ }^{21}$. La papyrologie juridique est très liée à la papyrologie historique, l'histoire des sociétés antiques ne pouvant s'écrire qu'en intégrant les données des droits antiques.

La papyrologie littéraire se consacre à des textes de nature très variée, poésie, tragédie, romans, œuvres historiques, géographiques, médicales, botaniques, mathématiques etc. Ils ont été rassemblés par Roger A. Pack ${ }^{22}$. Ce catalogue est revu et enrichi dans le "Mertens-Pack ${ }^{3}$ " mis en ligne par le Centre de Documentation Littéraire de l'Université de Liège (CEDOPAL) ${ }^{23}$. Ce domaine propre est lui-même lié à la papyrologie historique, d'abord parce qu'il procure des sources à l'historien, mais aussi par l'intérêt que procure leur étude en les replaçant - comme le prône Guglielmo Cavallo ${ }^{24}$ - dans leur «espace» et leur époque, dans leur production, leur circulation, et leur réception par leurs publics respectifs ${ }^{25}$.

La papyrologie herculanéenne constitue à elle seule une spécialité relevant de la papyrologie littéraire. Elle se fonde à la fois sur le traitement original requis pour ces rouleaux carbonisés issus des fouilles d'Herculanum en 1752, et par leur contenu spécifique, puisqu'il s'agit de textes philosophiques ${ }^{26}$.

La diversité de ces spécialisations papyrologiques justifie la diversité et la complémentarité des centres de recherches et d'enseignement ${ }^{27}$.

21 Cf. R.S. BAGNALL, Reading papyri, p. 11: "was Egypt "normal” or a world of its own?».

22 R.A. PACK, The Greek and Latin Literary Texts from Greco-Roman Egypt, 2 éd., Ann Arbor, 1965.

23 http://www.ulg.ac.be/facphl/services/cedopal/MP3/index.html

24 G. CAVAllo, P. FEDELI et A. GIARDINA, Lo spazio letterario di Roma antica, 5 vol., Rome, depuis 1989.

25 Cf. C. NICOLET, Mémoire, p. VI.

26 M. CAPASSO, Manuale di papirologia ercolanese, Naples, 1991.

$27 \quad \mathrm{La} \mathrm{IV}^{\mathrm{e}}$ section de l'École Pratique des Hautes Études, dédiée aux «Sciences historiques et philologiques», à Paris, possède, en 2004/2005, deux Directions d'études de papyrologie grecque, avec d'une part Joseph Mélèze Modrzejewski «Papyrologie et histoire des droits de l'Antiquité», et d'autre part Jean-Luc Fournet "Papyrologie grecque». En France, les chaires de Papyrologie, outre l'EPHE IV section, se concentrent à l'Université Paris IV (Sorbonne) avec le Professeur Alain Blanchard, et à Strasbourg (Université Marc Bloch) avec le Professeur Jean Gascou. La papyrologie documentaire est enseignée dans d'autres universités françaises, par exemple à l'Université Paris I Panthéon-Sorbonne, à l'Université Louis-Lumière (Lyon II), à l'Université de Reims Champagne-Ardenne. En Belgique, l'enseignement de la papyrologie (littéraire et/ou documentaire) se concentre à Bruxelles (Université Libre de Bruxelles), à Liège (Université de Liège) et à Louvain (Katholieke Universiteit Leuven). Dans l'espace francophone, il convient d'ajouter la tradition d'enseignement de la papyrologie à l'Université de Genève. Je remercie le Professeur Jean A. Straus (Université de Liège) pour l'aide qu'il m'a apportée pour cette rapide présentation de la papyrologie en Belgique. 


\section{La papyrologie documentaire: enjeux actuels}

Les enjeux principaux des recherches papyrologiques actuelles semblent pouvoir se ramener à trois axes, le renforcement de l'accessibilité de la discipline, la dynamisation des rencontres avec d'autres disciplines de l'Altertumswissenschaft, et le développement de problématiques nouvelles ou renouvelées.

\subsection{Le renforcement de l'accessibilité de la discipline: du papier à la Toile}

Les études papyrologiques se sont développées historiquement dans les centres universitaires riches en manuscrits papyrologiques. La géographie des congrès internationaux de papyrologues épouse de même - pour l'essentiel - celle de leurs lieux de conservation à travers le monde (Europe, Amérique du Nord, Égypte). Or ces lieux de conservation sont très variables suivant les pays. Si les universités allemandes possèdent des collections réparties dans de nombreux centres (Berlin, Beuron, Bonn, Brême, Cologne, Dresde, Erlangen, Francfort/Main, Fribourg/Brisgau, Gießen, Halle, Hambourg, Heidelberg, Jena, Munich, Leipzig et Wurzbourg), la France offre un tableau bien différent avec trois lieux de conservation, Strasbourg, Paris et Lille ${ }^{28}$.

La collection de papyrus de Strasbourg, la plus importante, est conservée à la Bibliothèque Nationale et Universitaire (BNUS). Toutes les langues/écritures de l'histoire de l'Égypte sont représentées, hiéroglyphique, hiératique, démotique, copte, grec (qui est majoritaire), latin et arabe. Les supports sont le papyrus (majoritaire), les ostraca, les tablettes et les étiquettes de momies. L'inventaire dénombre 10390 documents. Cette collection s'est constituée pour l'essentiel durant la période de l'annexion à l'Allemagne $(1871-1918){ }^{29}$. Les premiers achats furent effectués en 1899 à l'initiative de Richard Reitzenstein pour les textes grecs et de Wilhelm Spiegelberg pour les textes égyptiens. Les dernières acquisitions, effectuées par Jacques Schwartz, datent de 1968.

Les collections conservées à Paris sont réparties entre quatre institutions: l'Institut de Papyrologie de la Sorbonne (Université de Paris IV), le Musée du Louvre (Département des Antiquités égyptiennes pour les papyrus et les ostraca; Département des Antiquités grecques et romaines pour les tablettes), la Bibliothèque nationale de France (Département des manuscrits) et l'Institut de France. La collection la plus importante se trouve à l'Institut de Papyrologie de la

28 Pour les papyrus conservés dans d'autres villes, il ne s'agit que de pièces ne pouvant constituer des «collections», par exemple à Rouen.

29 A. MARTIN et P. HEILPORN, "La collection de papyrus de la Bibliothèque Nationale et Universitaire de Strasbourg", in Papyrus Collections World Wide, p. $77-$ 80. Site Internet: <http://www-bnus.u-strasbg.fr.> 
Sorbonne. Les langues/écritures sont majoritairement le grec, mais aussi le latin, le démotique, le copte et l'arabe. Les supports de l'écriture sont majoritairement le papyrus, le parchemin, le plomb et les ostraca. Les papyrus inventoriés comportent 2131 numéros. Cette collection s'est constituée à partir d'un fonds ancien remontant aux premières fouilles scientifiques tournées vers la recherche de papyrus en Égypte avec Pierre Jouguet, à Ghoran et Magdola, dans le Fayoum ${ }^{30}$.

La collection de l'Institut de Papyrologie et d'Égyptologie de l'Université de Lille est beaucoup plus modeste que les précédentes, Pierre Jouguet (1869-1949) ayant transporté une grande partie des cartonnages à Paris lorsqu'il fonda, en 1920, l'Institut de Papyrologie de la Sorbonne. Cette collection, qui s'est enrichie depuis, comporte des papyrus grecs et démotiques ${ }^{31}$.

La France se trouve dans une position médiane, entre les pays dont le fonds papyrologique est modeste (ainsi la Suisse où la collection genevoise - l'une des plus anciennes d'Europe - ne compte qu'un peu plus de 500 pièces, ou bien la Belgique où la collection bruxelloise compte quelques dizaines de documents en langue égyptienne et un inventaire de 416 documents grecs) 32 et les pays les mieux pourvus, en particulier l'Allemagne, l'Autriche, l'Égypte, les États-Unis d'Amérique, la Grande-Bretagne et l'Italie. L'université de Heidelberg a publié à ce jour 48069 papyrus documentaires!

La multiplication des publications et des sites papyrologiques sur la Toile permet un accès aisé à la documentation papyrologique, en constante augmentation.

Que de chemin parcouru depuis la publication du premier papyrus documentaire, la Charta Borgiana, par le Danois Niels I. Schow (1754-1830) en 1788 33! La comparaison, à dix ans de distance, entre la quatrième édition (Bulletin of the American Society of Papyrologists. Supplements 7/1992) et la cinquième (Bulletin of the Society of American Papyrologists. Supplements 9/2001), permet d'en prendre la mesure. Ce recueil indispensable qui tenait en 35 pages en comporte désormais $121^{34}$. Cette augmentation de volume ne résulte cependant

30 A. BLANCHARD, "Les collections de l'Institut de Papyrologie de la Sorbonne (Université de Paris IV)”, in Papyrus Collections World Wide, p. 71-73. Adresse Internet: papysorb@paris4.sorbonne.fr.

31 Je remercie le Professeur Alain Blanchard, directeur de l'Institut de Papyrologie de la Sorbonne, pour ces précieux renseignements.

32 Sur les collections genevoises, P. SCHUBERT, "Les papyrus de la Bibliothèque publique et universitaire de Genève", in Papyrus Collections World Wide, p. 241-243; sur la collection bruxelloise, A. MARTIN, "La collection de papyrus de BruxellesBrussel, Musées Royaux d'Art et d'Histoire (MRAH)", ibidem, p. 21-23.

33 N.I. SCHOW, Charta papyracea Graece scripta Musei Borgiana Velitris qua series incolarum Ptolemaidis Arsinoiticae in aggeribus et fossis operantium exhibetur, Rome, 1788. Cf. A. MARTIN, "En marge de la Charta Borgiana", Chronique d'Égypte 75 (2000), p. 118-125.

34 Le nombre de collaborateurs a également doublé. 
pas seulement du nombre plus élevé de corpus papyrologiques grecs et latins, mais aussi de son élargissement au démotique et au copte, ainsi que de l'accroissement des sections du recueil ${ }^{35}$. Mais un simple regard sur la Section VIII, la liste des éditions de papyrus documentaires grecs et coptes par année de publication permet de vérifier l'abondance et la régularité des nouveaux corpus. La seule collection des papyrus d'Oxyrhynchos s'est accrue, de 1992 à 2003, de neuf volumes (LIX à LXVIII) contenant 741 nouveaux textes documentaires et littéraires (numéros 3963 à 4704). Le dynamisme de l'activité éditoriale se vérifie aussi par la publication, depuis 1992, tous les trois ou quatre ans, de la Berichtigungsliste, qui enregistre les corrections apportées aux éditions de documents papyrologiques en grec ${ }^{36}$.

Ces nouveaux volumes de textes résultent tant de la publication de textes conservés dans les musées, instituts et universités, que de nouvelles trouvailles en Égypte même, qui ne cessent ni pour les papyrus ${ }^{37}$, ni pour les tessons, ni pour les tablettes, ni pour les étiquettes de momies.

La mise en ligne des corpus de papyrus documentaires par l'Université américaine Duke permet un accès "délocalisé» des sources, Duke Data Bank of Documentary Papyri (DDBDP) ${ }^{38}$ :

$<$ http://www.scriptorium.lib.duke.edu/papyrus/texts/DDBDP.html>

Cette base de données électronique doit être utilisée avec l'outil de recherche «Perseus Papyrological Resources»:

$<$ http://www.perseus.tufts.edu/Texts/papyri.html>

Pour les papyrus littéraires, il convient d'utiliser le Thesaurus Linguae Graecae $(T L G)^{39}$, le catalogue Mertens-Pack $3^{40}$, et la Leuven Database of Ancient Books $(L D A B)^{41}$.

35 La Checklist de 1992 comptait quatre sections: I. Papyrus, II. Ostraca, III. Corpora, IV. Séries; celle de 2001 comporte neuf sections: I. Papyrus, II. Ostraca et tablettes, III. Corpora, IV. Instrumenta, V. Séries, VI. Périodiques, VII. Informations relatives aux éditeurs, VIII. Liste des éditions de papyrus documentaires grecs et coptes par année de publication, IX. Actes de congrès internationaux.

36 Berichtigungsliste der Griechischen Papyrusurkunden aus Ägypten, I (1922), II (1933), III (1958), IV (1964), V (1969), VI (1976), VII (1986), VIII (1992), IX (1995), X (1998), XI (2002).

37 La construction du barrage d'Assouan a provoqué une montée de la nappe phréatique qui, combinée à l'extension des cultures et à l'urbanisation du pays, pourrait aboutir à une destruction des papyrus encore enfouis sous les sables: cf. C. GALAZZI, "Trouvera-t-on encore des papyrus en 2042?", in Proceedings of the 20th International Congress of Papyrologists (Copenhague, 23-29 août 1992), Copenhague, 1994, p. 131135.

38 Également disponible sur CD ROM, The Packard Humanities Institute. PHI 7 CD ROM, Los Altos CA, 1996.

39 <http://www.tlg.uci.edu/ $\sim \mathrm{tg} />$

40 Voir supra, note 23.

41 <http://www.tu.be/hit/hit.htm> 
La numérisation permet par ailleurs d'accéder à des photographies de papyrus (Duke University, Copenhague, etc.). Certaines publications renvoient directement à un site Internet sans reproduire la traditionnelle photographie, ainsi pour Roger S.Bagnall, Christina Helms et Arthur M.F.W. Verhoogt, Documents from Berenike. Volume I. Greek Ostraka from the 1996-1998 Seasons, Bruxelles, 2000, où les auteurs indiquent en préface que depuis janvier 2000 les tessons publiés sont visibles sur le site <http://www.columbia.edu/dlc/apis/berenike>

La mise en ligne concerne aussi des revues scientifiques, par exemple la Zeitschrift für Papyrologie und Epigraphik (Bonn): <http://www.uni-koeln.de/phil$\mathrm{fak} / \mathrm{ifa} / \mathrm{zpe} / \mathrm{index} \cdot \mathrm{html}>$

Le développement de l'outil électronique (CD Rom et Web) contribue au rayonnement de certains centres universitaires, qui ne possèdent pas ou peu de richesses papyrologiques, par exemple Liège ou Louvain (Leuven). La photographie avait déjà joué un rôle déterminant dans la constitution de "centres de données» sans fonds de manuscrits. Le Centre de documentation de Papyrologie Littéraire (CEDOPAL) de l'Université de Liège possède ainsi les photographies de tous les papyrus littéraires dispersés dans le monde. La photographie ne peut complètement remplacer l'accès à l'original, mais elle est un outil remarquable, surtout quand elle est de qualité, et si elle permet des agrandissements éclairants du manuscrit.

\subsection{La coopération avec les autres disciplines des Sciences de l'Antiquité:} les «recherches croisées»

La documentation papyrologique grecque et latine ne peut être utilisée seule pour construire un savoir total sur l'histoire de l'Égypte grecque, romaine et byzantine. travail.

Cette évidence s'est imposée dès la constitution des premiers instruments de

L'impérieuse nécessité pour le papyrologue d'utiliser également les sources épigraphiques de langue grecque s'exprime par exemple dans la conception du Sammelbuch der Griechischen Urkunden aus Ägypten dont le volume I fut publié par Friedrich Preisigke, à Berlin et Strasbourg, sous forme de fascicules entre 1913 et 1915. Ces volumes, au nombre de 24 en 2003, contenant à cette date 16340 documents, réunissent en effet les documents papyrologiques et épigraphiques édités dans les périodiques et les publications occasionnelles (Actes de colloques, de congrès, Mélanges etc.). L'enquête menée par Louis Robert sur les Cauniens en Égypte ne pouvait qu'être épigraphique et papyrologique ${ }^{42}$. La complémentarité entre ces deux types de sources se vérifie tant pour les régions ou les thèmes d'études

42 L. ROBERT, “À Caunos avec Quintus de Smyrne”, Bulletin de correspondance hellénique 108 (1984), p. 499-532 (en part. p. 526-532). 
où celles-ci sont substantielles que pour celles où elle sont déséquilibrées ${ }^{43}$. Le Fayoum peut fournir un exemple emblématique d'une documentation doublement abondante ${ }^{44}$. Alexandrie n'a pas livré de papyrus, perdus du fait de l'occupation permanente du site, mais les inscriptions alexandrines offrent alors leur richesse ${ }^{45}$. À l'inverse, la papyrologie fournit souvent des dossiers où les inscriptions font pâle figure, ainsi pour les «archives» de Zénon ${ }^{46}$.

Le papyrologue doit de même se tourner vers la totalité des sources littéraires, l'archéologie et la numismatique. L'édition de la description de l'Égypte, intégrée au livre XVII de la Géographie de Strabon, publiée en 1997, en est un bel exemple. Son ambition a été en effet de faire vivre cette fresque de l'Égypte sous Auguste sous «les regards croisés du géographe Strabon, de l'égyptologue (Jean Yoyotte), du diplomate (Stéphane Gompertz) et de l'helléniste (Pascal Charvet)» 47.

Les données de l'archéologie se révèlent également essentielles ${ }^{48}$. On sait l'apport récent de l'archéologie alexandrine pour notre connaissance de la ville

43 Cf. J. BINGEN, "Normalité et spécificité de l'épigraphie grecque et romaine d'Égypte", in Egitto e storia antica dall'ellenismo all'età araba, L.C RISCUOLO et G.G ERACI éd., Bologne, 1989, p. 15-35.

44 Cf. É. BERNAND, Recueil des Inscriptions grecques du Fayoum, 3 vol., Le Caire, 19751981.

45 Cf. F. KAYSER, Recueil des inscriptions grecques et latines (non-funéraires) d'Alexandrie

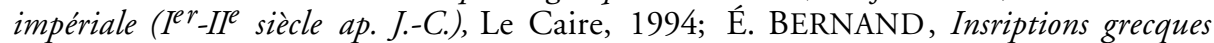
d'Alexandrie ptolémä̈que, Le Caire, 2001. Pour les inscriptions découvertes dans le port oriental: É. BERNAND, in F. GODDIO et alii, Alexandrie, les quartiers royaux submergés, Londres, 1998, p. 157-167.

46 Les noms du diøcète Apollonios et de Zénon de Caunos apparaissent vraisemblablement dans la stèle bilingue (hiéroglyphe/grec) SB I 5796 reprise par W.C LARYSSE et P.W. PESTMAN, Greek and Demotic Texts from the Zenon Archive (P.L. Batt. 20), P.W. PESTMAN éd., Leyde, 1980, Supplement: related texts, F.

47 J. YOYOTTE, P. CHARVET et S. GOMPERTZ, Strabon. Le voyage en Égypte. Un regard romain, Paris, 1997, p. 12. Cf. aussi p. 13 la liste des remerciements.

48 Les pionniers de la discipline viennent d'horizons très variés: Sir Flinders Petrie, qui inaugure en 1889-1890 les fouilles papyrologiques à caractère scientifique est un archéologue et un égyptologue, Bernard Grenfell et Arthur Hunt, les «Dioscures d'Oxford», ainsi que Pierre Jouguet sont des archéologues et des hellénistes, Ulrich Wilcken est un historien, Ludwig Mitteis un juriste, Friedich Preisigke "Telegraphendirektor» de la poste impériale allemande à Strasbourg, etc. Sur l'histoire de la discipline voir par ex. A. BATAILLE, "Papyrologie", p. 501-502, ou G. PURPURA, Diritto, papiri e scrittura, p. 32-39. Sur les premières fouilles françaises dans le Fayoum, cf. P.J OUGUET, "Fouilles du Fayoum", Bulletin de correspondance hellénique 25 (1901), p. 379-411 et P. JOUGUET - G. LEFEVRE, "Papyrus de Magdôla”, ibid., p. 95-128. Pierre Jouguet constate dans le premier article, p. 379-380, que «les Bédouins violateurs de tombeaux, (sont les) précurseurs et concurrents des antiquaires. Quiconque veut former une collection de papyrus doit pouvoir, non seulement fouiller, mais acheter». 
antique, et les résultats des travaux des archéologues italiens dans le Fayoum ${ }^{49}$. Les travaux récents sur les scènes nilotiques des mosaïques romaines ont contribué à une meilleure connaissance de l'univers monumental et architectural alexandrin 50. Mais il faut aussi rappeler qu'il existe pour certains sites une dissociation entre la richesse de la documentation papyrologique et la faiblesse des connaissances archéologiques, ainsi pour Philadelphie (Fayoum) ou pour Oxyrhynchos ${ }^{51}$.

L'apport de la numismatique a été valorisé par un certain regain d'études qui croisent, là encore, les sources papyrologiques 52.

L'absolue nécessité de connaître et d'utiliser les travaux des égyptologues travaillant sur les sources en langue égyptienne (hiéroglyphique, hiératique, démotique et copte) a été également reconnue dès la naissance de la science papyrologique grecque. Wilhelm Spiegelberg en a posé dès la fin du XIX ${ }^{e}$ siècle le principe en affirmant la nécessité de croiser les sources quelles que soient leurs langues ${ }^{53}$. Ce principe méthodologique a reçu le nom de Mischforschung, «recherche mixte». Sa mise en œuvre n'a pas toujours été aisée ${ }^{54}$. Heinz Heinen recommandait ainsi, lors d'un colloque international tenu à Bologne en 1987, de développer encore plus la collaboration scientifique entre hellénistes et égyptologues ${ }^{55}$. Des manuels d'études démotiques cités supra 56 permettent d'accéder à ces vastes champs disciplinaires toujours en progrès. L'intégration des sources démotiques et coptes à la Checklist de 2001 marque à ce égard un important tournant vers l'unification des outils de travail pour les spécialistes venus de différentes disciplines. La publication récente de différents dossiers bilingues

49 P. DAVOLI éd, L'archeologia urbana nel Fayyum di età ellenistica e romana, BologneLecce, 1997.

50 Cf. en dernier lieu M. MALAISE, "La signification des scènes nilotiques dans la culture romaine”, Chronique d'Égypte 78 (2003), p. 308-325.

51 Pour Oxyrhynchos, cf. H.I. AMER, "Le site de Bahnasa, relations extérieures", in La vallée du Nil et la Méditerranée. Voies de communication et vecteurs culturels, Actes du Colloque (5-6 juin 1998), S.H. AUFRÈRE éd., Montpellier, 2001, p. 3-9.

52 F. DUYRAT et O. PICARD, L'exception égyptienne. Production et échanges monétaires en Égyte hellénistique et romaine, Le Caire, à paraître.

53 Cf. par exemple cette publication de documents bilingues: W. SPIEGELBERG, Griechische und aegyptische Eigennamen aus Mumienetiketten der römischen Kaiserzeit, Leipzig, 1901.

54 Cf. à titre d'exemple le caractère souvent décisif des textes démotiques pour établir la chronologie: P.W. PESTMAN, Chronologie égyptienne d'après les textes démotiques (332 av. J.-C. - 453 apr. J.-C.), Papyrologiga Lugduno-Batava 15, Leyde, 1967.

55 H. HEINEN, "L'Égypte dans l'historiographie moderne du monde hellénistique", in Egitto e storia, p. 132-133.

56 Cf. note 5. 
permet de prendre la mesure de l'immense intérêt de cette "recherche croisée», qui doit être étendue à tous les champs des «Sciences de l'Antiquité» 57.

\subsection{Les problématiques de la papyrologie documentaire: les sciences historiques et juridiques}

Vouloir présenter un panorama des recherches papyrologiques actuelles n'est pas ici notre objectif. Il existe de fait de nombreuses publications permettant de suivre les travaux papyrologiques ${ }^{58}$. Les revues sont données par la Checklist. Une mention doit cependant être faite de la Bibliographie papyrologique, fondée en 1932 par Marcel Hombert, aujourd'hui rédigée par Georges Nachtergael et Alain Martin, qui réalise six envois annuels de 100 fiches. Il existe une version imprimée et une version électronique sur disquette 59 .

Si la publication de nouveaux documents est a priori indépendante des orientations thématiques de la recherche, celles-ci apparaissent clairement dans les domaines de recherche des papyrologues commentant et utilisant les papyrus édités.

Des travaux peuvent évidemment naître du désir d'approfondir l'étude d'un texte récemment publié. La publication du rouleau de Milan (P. Mil. Vogl. VII 309) a ainsi eu pour conséquence un très grand nombre de publications sur la poésie de Posidippe et l'épigramme grecque ${ }^{60}$. Pour la papyrologie documentaire, il faut citer le renouvellement de la question des politeumata avec la publication des papyrus grecs du politeuma juif d'Hérakléopolis ${ }^{61}$. Ces papyrus issus des

57 K. VANDORPe, P. Dryton. The Bilingual Family Archive of Dryton, Bruxelles, 2002. Sur l'apport des sources égyptiennes pour l'histoire de l'Égypte, cf. M.C HAUVEAU, L'Égypte au temps de Cléopâtre 180-30 av. J.-C., Paris, 1997.

58 L'Année philologique recense ces travaux sous les rubriques «Papyrologie», «Histoire de l'Égypte gréco-romaine», «Civilisation de l'Égypte gréco-romaine» et «Droit alexandrin et romano-égyptien».

59 La BP est éditée par la Fondation Égyptologique Reine Élisabeth (Bruxelles).

60 G. BASTIANINI , C. GAlaZZI et C. AUSTIN, Posidippo di Pella: Epigrammi (P. Mil. Vogl. VIII 309), Milan, 2001; C.A USTIN et G. BASTIANINI, Posidippi Pellaei quae supersunt omnia, Milan, 2002. Pour les travaux nés de cette publication cf. A.H ARDIE, "The Statue(s) of Philitas (P. Mil. Vogl. VIII 309 Col. X. 16-25 and Hermesianax fr. 7.75-78 P.)", Zeitschrift für Papyrologie und Epigraphik 143 (2003), p.27-36.

61 J.M.S. COWEY et K. MARESCH Urkunden des Politeuma der Juden von Herakleopolis (144/3-133/2 v. Chr. (P. Polit. Iud), Wiebaden, 2001. Cf. J.M ÉLĖZEMODRZEJEWSKI, "La Diaspora juive d'Égypte", in L'Orient méditerranéen de la mort d'Alexandre au $I^{e r}$ siècle avant notre ère, M.-Th.L ED INAHET éd., Nantes, 2003, p. 333-334; et ID., "La fiancée adultère. À propos de la pratique matrimoniale du judaïsme hellénisé à la lumière du dossier du politeuma juif d'Hérakléopolis (144/3133/2 avant n. è.)", dans J.-C.C OUVENHES et B. LEGRAS, Transferts culturels et politique à l'époque hellénistique, Actes de la journée d'études du 7 février 2004, Paris, à paraître. 
collections de Cologne, Munich, Heidelberg et Vienne, ont relancé le débat sur l'intégration des Juifs dans la société de l'Égypte ptolémaïque ${ }^{62}$. Pour l'Égypte romaine, on sait tout le profit que tirent les historiens des «archives d'Héroninos» qui sont - quelque part - pour le milieu du IIIe siècle de n. è. ce que sont les «archives de Zénon» pour le milieu du III ${ }^{\mathrm{e}}$ siècle av. n. è. ${ }^{63}$

Mais on peut aussi dégager des tendances liées aux grands domaines de recherche qui animent les travaux actuels des historiens et des juristes, ainsi des thèmes de la société multiculturelle ${ }^{64}$, de la violence endémique qui y règne ${ }^{65}$, ou des relations entre les hommes et les femmes ${ }^{66}$. Ces quelques thèmes, qui ne sont naturellement nullement exhaustifs de la recherche actuelle, sont cités ici, car il semble évident qu'ils peuvent s'enrichir d'une recherche "trans-période historique», qui tire sa légitimité de l'unité fondamentale de l'Histoire considérée comme une science globale. Dans cette perspective, les papyrologues doivent nouer un fructueux

62 Cf. S. HONIGMAN, "The Jewish Politeuma at Herakleopolis", Scripta Classica Israelica 21 (2002), p. 251-266; K.M ARESCH et J.M.S. COWEY, "A Recurrent Inclination to Isolate the Jews from their Ptolemaic environment", Scripta Classica Israelica 22 (2003), p.307-310.

63 La première étude synthétique est celle de D. RATHBONE, Economic Rationalism and Rural Society in Third-Century A.D. Aegypt. The Heroninos Archive and the Appianus Estate, Cambridge, 1991. Cf. E. JAKAB, "Fixierte Preise für die Vermaktung des Weins im Heroninos-Archiv", Münsterische Beiträge zur antiken Handelgeschichte 22 (2003), p. 1-26.

64 Cf. B. LEGRAS, L'Égypte grecque et romaine, Paris, Armand Colin, U-Histoire, 2004, p.162-184. La question de la société multiculturelle de l'Égypte touche particulièrement aux univers religieux: cf. en particulier D. FRANKFURTER, Religion in Roman Egypt. Assimilation and Resistance, Princeton, 1998 (ce titre n'a malencontreusement pas été imprimé dans la Bibliographie de mon livre).

65 Cf. pour l'Égypte romaine, R. BAGNALL, "Official and Private Violence in Roman Egypt", The Bulletin of the American Society of Papyrologists 26 (1989), p. 201-216, et R.A LSTON, "Violence and Social Control in Roman Egypt", in Proceedings of the $20^{\text {th }}$ International Congress of Papyrologists (Copenhague, 23-29 août 1992), Copenhague, 1994, p. 165-176.

66 Cf. I. BIEZUNSKA-MALOWIST, "Les recherches sur la condition de la femme grecque en Égypte grecque et romaine, hier et aujourd'hui”, Antiquitas 18 (1993), p.15-22 (Acta Universitatis Wratislaviensis, Wroclaw, $\mathrm{n}^{\circ} 1435$ ); J.R OWLANDSON (éd.), Women and Society in Greek \& Roman Egypt. A Sourcebook, with the contribution of R. BAGNALL, A.B OWMAN, W. ClarysSE, A.E. HANSON, D. HOBSON, J.K EENAN, P. VAN MINNEN, D. RATHBONE, Cambridge, 1998. Cf. aussi B.L EGRAS, "Le corps violenté des femmes dans l'Égypte ptolémaïque d'après la documentation papyrologique", Cahiers du Centre Gustave-Glotz 10 (1999), p. 225234; "L'adoption en droit hellénistique, d'après les papyrus grecs d'Égypte", in Parenté, sexe et genre dans le monde grec, de l'Antiquité à l'âge moderne (Colloque international de Volos, 18-21 juin 2003), Bordeaux (sous presse) et "Masculin/féminin dans le droit familial d'après la documentation papyrologique grecque d'Égypte”, in Genre et Antiquité (à paraître). 
dialogue avec les historiens travaillant sur des problématiques proches ou semblables dans d'autres sociétés humaines de l'Antiquité et d'au-delà 67.

\section{Conclusion}

Selon une phrase traditionnellement prêtée à Theodor Mommsen, mais qu'il faut restituer à son véritable auteur, Ludwig Mitteis ${ }^{68}$, le $\mathrm{XX}^{\mathrm{e}}$ siècle devait être celui de la papyrologie, le XIXe siècle ayant été celui de l'épigraphie ${ }^{69}$. Si les papyrologues tournés vers l'histoire et le droit savent valoriser cette double ouverture - d'une part vers les autres sciences et techniques de l'Altertumswissenschaft, et d'autre part vers les problématiques stimulantes des sciences historiques et juridiques -, cette spécialité, fondée sur des sources dont le nombre et la richesse ne cessent de s'accroître, doit pouvoir s'inscrire encore plus fermement dans l'univers scientifique contemporain, et susciter de nouvelles vocations ${ }^{70}$.

\section{Annexe: Manuels de papyrologie}

MITTEIS (Ludwig) et WILCKEN (Ulrich), Grundzüge und Chrestomathie der Papyruskunde, 4 vol., Leipzig-Berlin, 1912.

SCHUBART (Wilhelm), Einführung in die Papyruskunde, Berlin, 1918.

67 De même que la chronique de J. MÉLĖZE-MODRZESEWSKI publiée dans la Revue historique de Droit français et étranger, celle de J. HENGSTL traite de la papyrologie juridique grecque replacée dans l'ensemble des Droits de l'Antiquité, en dernier lieu: "Juristische Literaturübersicht 1999-2001 (mit Nachträgen aus der vorausgegangenen Zeit)", Journal of Juristic Papyrology 33 (2003), p. 273-368. Cf. aussi des anthologies qui intègrent la documentation papyrologique au sein d'autres sources, par exemple: J.-M. BerTRAND, L'hellénisme 323-31 av. J.-C. Rois, cités et peuples, Paris, 1992; R.S.B AGNALL et P. DEROW, The Hellenistic Period: Historical sources in Translation, Malden, Mass.-Oxford, 2004.

68 Cf. A. BATAILlE, "Papyrologie", p. 512.

69 A. MARTIN, "Das Jahrhundert der Papyrologie", Archiv für Papyrusforschung 46 (2000), p.1-2. Ludwig Mitteis a prononcé cette phrase le 5 avril 1900 devant l'assemblée des historiens allemands réunis à Halle.

70 La publication d'anthologies de papyrus traduits est à cet égard vraiment indispensable. Cf. parmi les travaux scientifiques récents: X. DURAND , Des Grecs en Palestine au III siècle avant Jésus-Christ. Le dossier syrien des archives de Zénon de Caunos (261-252), Paris, 1997 ; P. SCHUBERT (éd.), Vivre en Égypte gréco-romaine, Vevey, 2000. Il n'existe pas encore - à ce jour - de Manuel de papyrologie en français. Mais l'écriture de tels ouvrages est actuellement en projet. 
CALDERINI (Aristide), Manuale di papirologia, Milan, 1938 (trad. espagnole: Tratado de Papirologia, Barcelone, 1963).

PEREMANS (Willy) et VERGOTE (Joseph), Papyrologisch Handboek, Louvain, 1942.

D'ORS (Alvaro), Introducción ad estudios de los documentos del Egipto romano, Madrid, 1948.

Turner (Eric G.), Greek Papyri. An introduction, 2e éd., Oxford, 1980 (trad. italienne: Papiri greci, Rome, 1984).

GALLO (Italo), Avviamento alla papirologia greco-latina, Naples, 1983 (trad. anglaise: Greek and Latin Papyrology, Londres, 1986).

FIKHMAN (Itzhak F.), Introduction à la papyrologie documentaire (en russe), Moscou, 1987.

MONTEVECCHI (Orsolina), La papirologia, nouvelle éd., Milan, 1988.

MANDilaras (Basile G.), Papuroi kai Papurologia (en grec moderne), Athènes, 1994.

MigLiardi ZINGALE (Livia), Introduzione allo studio della Papirologia giuridica, $2^{e}$ éd., Turin, 1994.

PeSTMAN (Pieter W.), The New Papyrological Primer, 2e éd., Leyde, 1994.

RUPPRECHT (Hans-Albert), Kleine Einführung in die Papyruskunde, Darmstadt, 1994 (trad. italienne : Introduzione alla Papirologia, Turin, 1999).

Bernard LEGRAS

Université de Reims

4, rue de Chantilly

F-75009 Paris

belegras@club-internet.fr 